\title{
Onabotulinum toxin $A$ in the treatment of chronic migraine: patient selection and special considerations
}

This article was published in the following Dove Press journal: Journal of Pain Research

29 September 2017

Number of times this article has been viewed

\author{
Piero Barbanti' \\ Patrizia Ferroni ${ }^{2}$ \\ 'Headache and Pain Unit, Department \\ of Neurological, Motor and \\ Sensorial Sciences, ${ }^{2}$ Department of \\ Human Sciences and Quality of Life \\ Promotion, San Raffaele Roma Open \\ University, IRCCS San Raffaele Pisana, \\ Rome, Italy
}

\begin{abstract}
Discovered by serendipity, onabotulinum toxin A (BoNT-A) is the only US Food and Drug Administration-approved treatment for the prevention of chronic migraine (CM), one of the most disabling and burdensome human conditions. Its efficacy, safety and tolerability, proved by the largest and longest migraine therapeutic trial (the Phase III Research Evaluating Migraine Prophylaxis Therapy program [PREEMPT]), have been replicated by various real-life studies also in the presence of medication overuse. The benefit of BoNT-A prophylaxis is likely due to its ability to counteract peripheral and central nociceptive sensitization through reversible chemical denervation of pericranial sensitive afferents. Its efficacy increases considerably over time during long-term treatments, significantly varying among patients. The present review focuses on the state-of-the art of current knowledge on putative instrumental, biochemical and clinical predictors of BoNT-A responsiveness, outlining the need for a thorough characterization of the full phenotypic migraine picture when trying to predict good responders. Available evidence suggests that disentangling the BoNT-A responsiveness puzzle requires 1) a reappraisal of easy-obtainable clinical details (eg, site and quality of pain, presence of cranial autonomic symptoms), 2) a proper stratification of patients with CM according to their headache frequency, 3) the evaluation of potential synergistic effects of concomitant prophylaxis/treatment and 4) a detailed assessment of modifiable risk factors evolution during treatment.
\end{abstract}

Keywords: chronic migraine, onabotulinum toxin A, prophylaxis, treatment responder, patient selection, disability

\section{Introduction}

Chronic migraine (CM), a headache occurring on $>15$ days/month (with migraine characteristics on $>8$ days/month) for at least 3 months, affects $2-3 \%$ of the general population and causes extreme disability. ${ }^{1,2} \mathrm{CM}$ evolves from episodic migraine in susceptible individuals through a sequence of mechanisms - still largely unknown including central sensitization, reduced descending pain inhibitory control and cortical hyperexcitability. ${ }^{3-8}$ To date, onabotulinum toxin A (BoNT-A) is the only treatment selectively approved for CM prophylaxis. ${ }^{9}$ Albeit a growing number of experimental and clinical studies have increased our comprehension on its mechanisms of action and therapeutic benefits in CM, efforts are still needed to identify responders and to improve the design of clinical trials for an easier translation of scientific data into clinical daily practice. ${ }^{10}$

The present review will focus on the state-of-the art of current knowledge on putative instrumental, biochemical and clinical predictors of BoNT-A responsiveness, exploring unmet needs and suggestion on future directions.
Correspondence: Piero Barbanti Headache and Pain Unit, Department of Neurological, Motor and Sensorial Sciences, IRCCS San Raffaele Pisana, Via della Pisana 235, 00163 Rome, Italy Tel +3906 52252477

Fax +3906 52252414

Email piero.barbanti@sanraffaele.it 


\section{BoNT-A: chemistry}

BoNT-A is one of the various neurotoxins produced by Clostridium botulinum, a gram-positive rod-shaped anaerobic bacterium, responsible for botulism following ingestion of contaminated canned or home-made foods. ${ }^{11}$

BoNT-A is a $900 \mathrm{kDa}$ complex made of a $150 \mathrm{kDa}$ toxic part and $750 \mathrm{kDa}$ accessory hemagglutinin and nonhemagglutinin proteins supposed to maintain BoNT-A stability. ${ }^{12}$ The toxic fraction is composed by a $100 \mathrm{kDa}$ heavy chain linked to a $50 \mathrm{kDa}$ light chain with a disulfide bridge. Once the polysialoganglioside protein acceptor in the outer side of the presynaptic ending via the heavy chain is recognized, BoNT-A is internalized into synaptic vesicles and light chain released into the cytosol. Free light chain, in turn, is responsible for the cleavage of synaptosomal-associated protein of $25 \mathrm{kDa}$ (SNAP-25), whose integrity is essential for the full fusion of synaptic vesicles with plasma membrane and neurotransmitter release (Figure 1). In fact, the interaction between SNAP-25 with vesicle-associated membrane protein/synaptobrevin and syntaxin (which constitute the soluble n-ethylmaleimide-sensitive factor attachment protein receptor complex) controls delivery of ion channels, neurotransmitters and receptors from presynaptic vesicles. ${ }^{13}$

\section{BoNT-A in headache disorders}

BoNT-A represents the most popular plastic surgery procedure worldwide. Its potential usefulness in headache therapy was serendipitously discovered in 1998 by a plastic surgeon, Dr. William Binder, who noted that BoNT-A treatment of hyperfunctional upper facial lines (in forehead, temporal and/or glabella regions) was associated with a significant improvement of concomitant "migraine or chronic headache pain". ${ }^{14}$ Since then, the toxin has been tested in the prevention of different episodic and chronic headaches, providing at first disappointing results.

\section{Tension type headache}

BoNT-A does not work in chronic tension type headache (CTTH) prophylaxis. A randomized placebo-controlled trial (RCT) on 300 patients demonstrated the superiority of

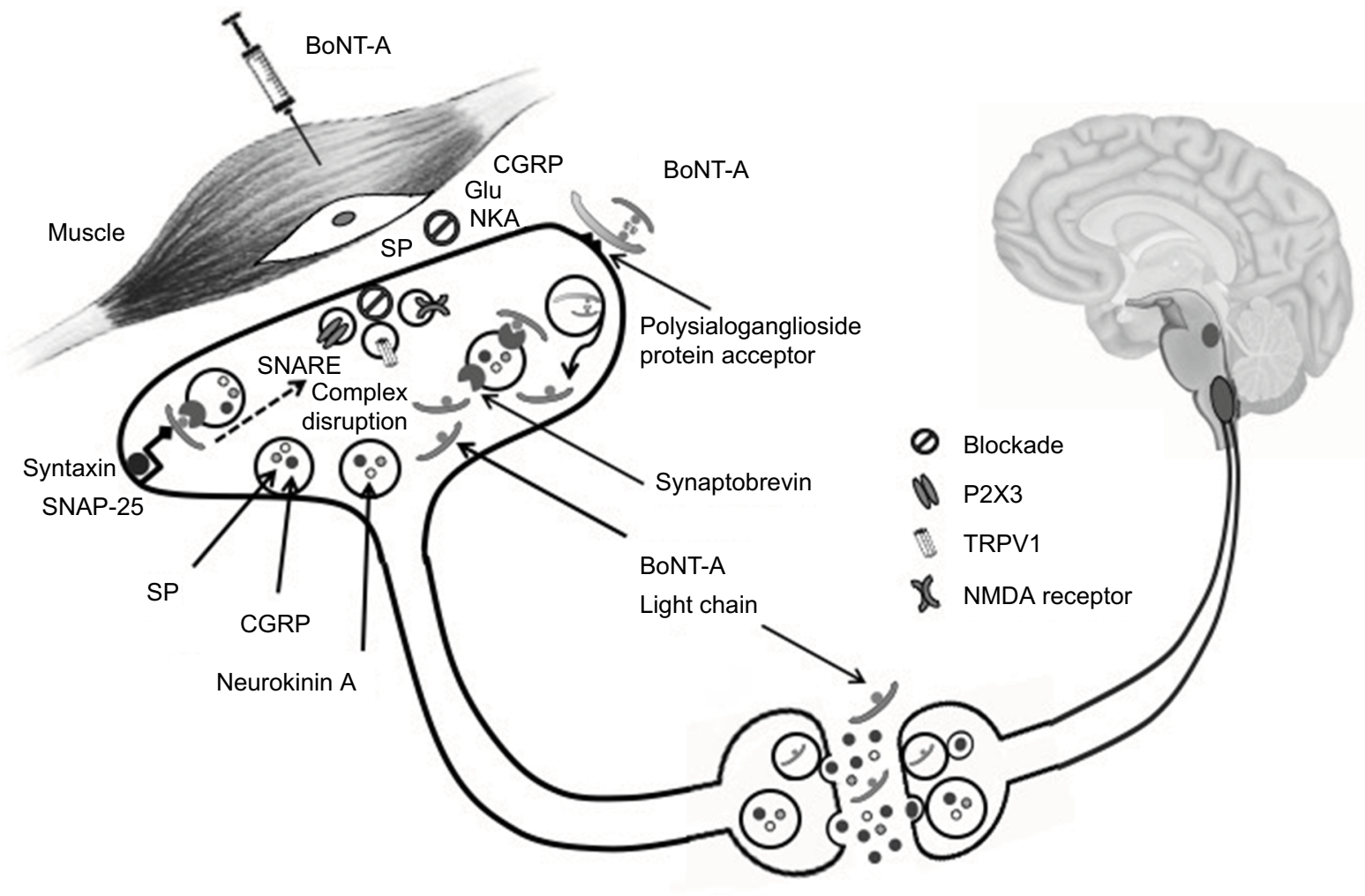

Figure I BoNT-A in migraine: putative mechanisms of action.

Notes: BoNT-A induces a chemical denervation, which reverts peripheral sensitization and, indirectly, central sensitization. BoNT-A injection in pericranial muscles blocks neuropeptide (substance $\mathrm{P}, \mathrm{CGRP}$ ) and neurotransmitter (Glu) release from peripheral trigeminal sensory nerve endings. BoNT-A also deranges the translocation to nerve ending plasma membrane of NMDA glutamate receptor, TRPVI and P2X3 purinoreceptors. A still controversial hypothesis suggests that BoNT-A could also act centrally, being transferred to second-order nociceptive neurons via retrograde axonal transport and transcytosis.

Abbreviations: BoNT-A, onabotulinum toxin A; CGRP, calcitonin gene-related peptide; Glu, glutamate; NMDA, N-methy-D-aspartate; TRPVI, transient receptor potential vanilloid I; NKA, Neurokinin A; SP, substance P; SNARE, soluble n-ethylmaleimide-sensitive factor attachment protein receptor; SNAP-25, synaptosomal-associated protein of $25 \mathrm{kDa}$. 
placebo versus BoNT-A (dose range 50-150 U, 10 injections in five muscular groups for each side) in monthly headache day reduction at day 60 (primary endpoint), even though BoNT-A was better at day 90 (secondary endpoint). ${ }^{15}$

\section{Chronic daily headache}

The prophylactic effect of BoNT-A was tested in a RCT in chronic daily headache. ${ }^{16}$ After screening placebo responder from non-responders, 355 patients with "headaches on more than 15 day of the 30-days baseline period" were treated with BoNT-A (mean dose 190 U, range 105-260 U) using a "follow the pain" approach with a variable number of injection sites over 7 muscles (frontal/glabella, occipitalis, temporalis, masseter, trapezius, semispinalis and splenius capitis). ${ }^{17}$ However, at day 180 , the difference in headache-free days between active drug and placebo was not significant (6.7 vs 5.2). Unfortunately, the study was biased by an obsolete diagnosis, which did not allow to differentiate patients affected by CM from those with CTTH. ${ }^{18}$

\section{Episodic migraine}

Controlled studies disproved the initial hypothesis that BoNT-A treatment could prevent episodic migraine, as suggested by a pioneering open study. ${ }^{19}$ A meta-analysis of 8 RCTs comparing the efficacy of pericranial injections of BoNT-A (dose range 7.5-260 U) versus placebo in the prophylaxis of episodic migraine on a total of 1.601 patients revealed no significant difference in the overall treatment effect size on migraine frequency for BoNT-A over placebo at 30, 60 and 90 days. ${ }^{20}$

\section{Trigeminal autonomic cephalgias and cranial neuralgias}

BoNT-A could be useful in the treatment of intractable chronic cluster headache, hemicrania continua and occipital neuralgia, according to the findings of small uncontrolled studies. ${ }^{21-26}$ In trigeminal neuralgia, BoNT-A outperformed placebo for responders' proportion, daily paroxysm frequency and pain intensity, as reported by a systematic review and a meta-analysis of 4 RCTs including 178 patients. ${ }^{27}$

\section{Chronic migraine}

The formulation of more precise chronic headache diagnostic criteria and the setup of ad hoc-designed clinical trials ultimately led to the demonstration that BoNT-A is effective in CM prophylaxis. ${ }^{28-31}$

\section{The PREEMPT study}

The efficacy and safety of BoNT-A in CM prevention were explored in the Phase III Research Evaluating Migraine
Prophylaxis Therapy (PREEMPT) program consisting of 2 large parallel RCTs: the PREEMPT 1 study, carried out at 56 North American sites on 679 patients, and the PREEMPT 2 study, enrolling 705 patients recruited at 50 North America and 16 European sites. ${ }^{30,31}$ Both trials shared the same protocol design characterized by a 24-week randomized, doubleblind (DB) phase followed by a 32-week open-label (OL) phase. Patients were randomly treated with BoNT-A (155 U) or placebo (ratio 1:1) in 31 fixed-site, fixed-dose injections across 7 head/neck muscles every 12 weeks for 5 cycles. At investigator's discretion, additional $40 \mathrm{U}$ was given in the temporalis, occipitalis or trapezius muscles using a followthe-pain treatment paradigm.

The PREEMPT 2 study reached the primary efficacy endpoint (change from baseline in frequency of headache episodes at week 24 : -9.0 vs $-6.7 ; p<0.001$ ) unlike the PREEMPT 1 (change from baseline in headache day frequency at week 24: -5.2 vs $-5.3 ; p=0.344)$. In addition, BoNT-A outperformed placebo in 2 out of the 4 pre-specified secondary endpoints in the PREEMPT 1 study (frequency of headache and migraine days) and in all 5 secondary endpoints in the PREEMPT 2 study (frequency of headache and migraine days, headache episodes, cumulative total headache hours on headache days, $\%$ of patients with Headache Impact Test (HIT)-6 score $>60$ ). Most patients overused analgesics. At week 24 , no significant reduction in acute medication intake was observed in both studies, although a post hoc analysis documented a triptan use reduction (PREEMPT 1: -3.3 vs $-2.5 ; p=0.023$; PREEMPT 2 : -3 vs $-1.7 ; p<0.001)$. Adverse events, usually of mild or moderate severity, occurred more frequently in patients treated with BoNT-A than in placebotreated patients (PREEMPT 1: 59.7\% vs 46.7\%; PREEMPT 2: $65.1 \%$ vs $56.4 \%) .{ }^{30,31}$

Pooled analyses of the 56-week PREEMPT clinical program revealed that the early BoNT-A treatment was more effective than the late one. In fact, chronic migraineurs receiving BoNT-A in both the double-blind and the openlabel phases $(B / B)$ reported a greater reduction in headache day frequency at week 56 (primary endpoint) than those treated with placebo during the double-blind phase $(\mathrm{P} / \mathrm{B})$ $(-11.7$ vs $-10.8 ; p=0.019)$, showing also a greater reduction in migraine days ( $p=0.018$ ), moderate/severe headache days ( $p=0.027$ ) and cumulative headache hours on headache days $(0=0.018){ }^{32}$ Nevertheless, at the end of OL (when all had received the active treatment), both $\mathrm{B} / \mathrm{B}$ and $\mathrm{P} / \mathrm{B}$ significantly improved in all efficacy measures compared to baseline, demonstrating a continued improvement over repeated BoNT-A treatments cycles. BoNT-A was safe and well tolerated as 
indicated by the high proportion of patients completing the 56-week study $(72.6 \%)$ and the very low discontinuation rate due to adverse events $(4.6 \%)$. $^{32}$

\section{PREEMPT study sub-analysis}

The PREEMPT program, the largest and longest migraine prophylactic trial, gave rise to several sub-analyses.

\section{Safety and tolerability}

A good safety and tolerability profile was highlighted by a pooled analysis of 5 trials (including the 2 PREEMPT studies and 2 previous exploratory phase II trials) with multiple BoNT-A treatments (up to 5 cycles) using a mean $163 \mathrm{U}$ dose (range 75-260 U). ${ }^{33}$ Adverse events, most commonly neck pain and muscle weakness, were mild or moderate in intensity and occurred in $72.9 \%$ of patients (placebo $56.8 \%$ ). Serious adverse events were reported by $5.4 \%$ of patients receiving the active drug and $3 \%$ of placebo-treated patients.

\section{Proportion of responders per treatment cycle}

Patients not responding to the first BoNT-A treatment cycle may well respond to the second or third one. The pooled PREEMPT data showed, in fact, that albeit half of the patients responded to the first BoNT-A treatment cycle ( $\geq 50 \%$ reduction in headache days: $49.3 \% ; \geq 50 \%$ reduction on cumulative headache hours: $54.2 \%$; total HIT-6 $\geq 5$-point improvement: $56.3 \%$ ), more than 1 out of 10 responded to the second $(11.3 \%, 11.6 \%$ and $14.5 \%$, respectively) and others to the third cycle ( $10.3 \%, 7.4 \%$ and $7.7 \%$, respectively), probably due to an inter-individual variability in time needed to reverse the central sensitization. ${ }^{34}$

\section{Presence of medication overuse at baseline}

BoNT-A was proven to be effective also when considering the subgroup of migraineurs with medication overuse (MO) at baseline. ${ }^{35}$ At week 24, these patients had a meaningful improvement in headache and migraine days $(p<0.001)$, cumulative headache hours on headache days $(p<0.001)$, headache episodes $(p=0.028)$, migraine episodes $(p=0.018)$ and proportion of patient with HIT-6 score $>60(p<0.001)$. Conversely, the acute analgesic intake was not significantly modified. It is worth mentioning that BoNT-A overperformed placebo in the proportion of patients who showed a sustained shift from MO to no MO at 3 and 6 months $(p=0.002){ }^{35}$

\section{Patients receiving all PREEMPT treatment cycles}

Two-thirds (72.6\%) of patients enrolled in the PREEMPT studies were treated with BoNT-A in all the 5 treatment cycles $(\mathrm{B} / \mathrm{B})$, whereas $27.4 \%$ received BoNT-A in only 3 cycles $(\mathrm{P} / \mathrm{B})$, during the $\mathrm{OL} .{ }^{36} \mathrm{~B} / \mathrm{B}$ continued to show lower frequency of headache days $(p=0.035)$, migraine days $(p=0.038)$ and moderate/severe headache days $(p=0.042)$ at week 56 compared to $\mathrm{P} / \mathrm{B}$, with no plateau effect, in line with progressive and addictive therapeutic benefits over time. ${ }^{36}$

\section{Improvement in migraine impact and quality of life}

Migraine impact on quality of life, measured by HIT-6 and Migraine-Specific Quality of Life Questionnaire (MSQ), continued to improve during long-term BoNT-A treatment. In the PREEMPT program, B/B maintained the HIT- 6 and MSQ benefits obtained in the DB period also during the $\mathrm{OL}$, whereas $\mathrm{P} / \mathrm{B}$ reached $\mathrm{B} / \mathrm{B}$ improvements at the end of the OL. ${ }^{37}$

\section{Real-life studies}

The benefits of BoNT-A in CM prophylaxis also emerged in a number of investigations carried out in real-life settings. ${ }^{38-44}$ The largest post-marketing, real-life, prospective studies on BoNT-A prophylaxis in CM were performed in the UK. The first, considering 254 patients attending a headache center, basically confirmed the efficacy and tolerability data of the PREEMPT studies. ${ }^{45}$ Headache days were reduced by $>50 \%$ in $32 \%$ and $>75 \%$ in $14 \%$ of patients, while migraine days were reduced by $>50 \%$ in $50 \%$ and $>75 \%$ in $24 \%$ of cases, demonstrating a significant increase in crystal clear days (no pain at all), lower analgesic assumption, reduced work absenteeism and improved quality of life. Of note, patients included in this trial had more severe conditions than those of the PREEMPT studies because almost all (94.4\%) had been previously treated with $>3$ preventative treatments and had a higher baseline headache frequency. ${ }^{45}$ The same research group confirmed these findings in a larger outpatient population (434 patients), emphasizing the same BoNT-A responsiveness (primary and secondary endpoints) in patients with MO (50.3\%) who outperformed those without MO in terms of 2-fold and 3-fold increase in headache-free days. ${ }^{46}$

\section{Long-term studies}

As CM is a chronic condition, an important question is how long should BoNT-A treatment last. There is evidence prompting its long-term use ( $>1$ year) in CM. BoNT-A benefits seem sustained, as documented by a study reporting that $74.2 \%$ of the 108 responders during the first year still respond to the treatment at 2 years and, among them, $90 \%$ continue to benefit for longer periods ${ }^{47}$ In $60 \%$ of cases, the attempt to postpone BoNT-A administration to 4 months seemed unsuccessful, 
leading to prefer the classic quarterly injection program. On the other hand, $40 \%$ of patients had benefit even with less frequent BoNT-A inoculation. ${ }^{47}$ Long-term treatment with doses ranging from 155 to 195 UI may also significantly reduce $\mathrm{MO}(61.9 \%)$ and induce discontinuation of concomitant prophylaxis (CP) in almost half of patients $(48.8 \%)^{42}$

Long-term trials specifically focusing on $\mathrm{MO}$ pointed out that BoNT-A benefit is not only sustained but even increased over repeated administrations. ${ }^{48-50}$ Extending the treatment from 6 to 18 months induced a further significant improvement of headache index (12\%), analgesic consumption (41\%), pain intensity (22\%), and headache-related disability and quality of life scores. ${ }^{48}$ In addition, 8 BoNT-A treatments over 24 months (especially at the dose of $195 \mathrm{U}$ ) caused a significant reduction in headache days, migraine days, medication intake days and HIT-6 scores. ${ }^{49}$

\section{Comparative trials}

BoNT-A proved effective as topiramate (TPM), a wellestablished prophylactic drug for CM. ${ }^{51}$ Two small RCT trials reported similar clinical benefits in patients randomized to BoNT-A (2 treatment cycles at baseline and month 3; maximum dose: $200 \mathrm{U}$ ) plus oral placebo or to TPM $100 \mathrm{mg}$ (up to $200 \mathrm{mg}$ ) plus saline injections. ${ }^{52,53}$ BoNT-A provoked less adverse events and less discontinuation than TPM only in the first pilot study. ${ }^{52}$

\section{Studies on specific migraine subpopulations CM with comorbid depression}

Depression is frequently comorbid with $\mathrm{CM}$ and may represent a significant obstacle to its successful treatment. An open-label study on a small sample (32 patients) of CM subjects with comorbid depression hinted that BoNTA (given at the dose of $155 \mathrm{U}$ following the PREEMPT paradigm) not only reduced headache frequency, severity and disability but also improved depression and anxiety (through direct or indirect mechanism). ${ }^{54}$ These findings, however, were not replicated by subsequent, larger, observational studies. ${ }^{43,48}$

\section{Cervical dystonia with concomitant migraine}

Headache attributed to craniocervical dystonia, as currently classified, is a very rare event. ${ }^{1,55}$ However, migraine may be comorbid in patients with cervical dystonia. In these subjects, high BoNT-A doses (up to $175 \mathrm{U}$ for dystonia plus up to 125 $\mathrm{U}$ for migraine) significantly reduced headache days, but not migraine days, at day 180 compared to baseline $(-4.38+7.99$ from a baseline of $15.33+6.7$ days; $p=0.0178) .{ }^{56}$

\section{Putative mechanism of action of BoNT-A in headache}

How BoNT-A acts in migraine prevention is still debated (Figure 1) ${ }^{57} \mathrm{~A}$ number of experimental cues suggest that BoNT-A disrupts peripheral and, indirectly, central sensitization by inducing a reversible chemical denervation of peripheral nociceptive endings through the following hypothetical mechanisms of action:

Blockade of the release from peripheral trigeminal sensory nerve endings of neuropeptides (substance $\mathrm{P}$, calcitonin gene-related peptide [CGRP]) and/or neurotransmitters (glutamate)..$^{58,59}$

Derangement of nociceptive receptors and ion channels translocation to synaptic plasma membrane (namely, $\mathrm{N}$-methy-D-aspartate glutamate receptor, transient receptor potential vanilloid 1 and $\mathrm{P} 2 \mathrm{X} 3$ purinoreceptors). ${ }^{59-61}$

It is worth mentioning that some researchers hypothesized that BoNT-A could act also centrally, via retrograde axonal transport and transcytosis to second-order nociceptive neurons. ${ }^{62}$

\section{How to identify CM patients responsive to BoNT-A?}

\section{Neuroimaging}

BoNT-A responders could have distinctive brain morphological and functional properties as suggested by a functional magnetic resonance imaging study in patients with $\mathrm{CM}$ revealing "a significant cortical thickening of right primary somatosensory cortex, anterior insula, left superior temporal gyrus and pars opercularis" in responders compared to nonresponders..$^{63}$ However, whether such brain changes - occurring in areas corresponding to sensory face representation and autonomic/interoceptive/emotional processing - are BoNT-A-induced or rather reflect pre-existing premorbid brain condition is uncertain. ${ }^{63}$

\section{Biochemistry}

Increased plasma levels of CGRP (the marker of trigeminal activation) and vasoactive intestinal peptide (VIP, the marker of parasympathetic activation) could distinguish BoNT-A responders from non-responders. ${ }^{64}$ In particular, CM patients with a CGRP level above the cutoff value of $72 \mathrm{pg} / \mathrm{mL}$ seem to have 28 times higher probability of being BoNT-A responders. ${ }^{64}$ Moreover, elevated baseline CGRP plasma concentrations are significantly decreased 1 month after treatment (from 76.85 to $52.48 \mathrm{pg} / \mathrm{mL} ; p=0.003$ ) only in BoNT-A responders, suggesting a BoNT-A-induced reversal of peripheral nociceptive sensitization in such patients. ${ }^{65}$ 
The aforementioned studies represent the first demonstration that biochemical parameters may not only identify $\mathrm{CM}$ but also detect responsive subgroups. ${ }^{64-66}$ Nevertheless, as commonly happens in clinical trials, in spite of a quite detailed description of patient treatments and comorbidities, no clinical details on migraine are provided by these studies, if one excludes the simple diagnostic distinction between the episodic and chronic form. In addition, the introduction of the sub-categories of "moderate" and "excellent" responders (which identify patients improving headache frequency between $33-66 \%$ and $>66 \%$ post-treatment, respectively), although innovative, could be misleading, increasing the proportion of "true" CGRP-responders. ${ }^{65}$

\section{Clinical features}

We suggest that an easy obtainable method to foresee the response to BoNT-A (and also other trigeminal-targeted treatment) might be to check some too often neglected migraine simple clinical features: where the headache is? How the pain is like? ${ }^{10}$

\section{Migraine attack-associated features: unilateral cranial} autonomic symptoms

A considerable proportion of migraine patients (1 out of 3 ) has unilateral autonomic symptoms (UAs: eyelid edema, tearing, nasal congestion, etc.) during the attack. ${ }^{67,68}$ These migraineurs (UAs+), clinically characterized by a more severe and strictly unilateral headache compared to general migraine population, show a pain topography, which traces the distribution of the ophthalmic branch of the trigeminal nerve, in line with a very intense peripheral nociceptive sensitization.$^{68}$ Such a heavy peripheral nociceptive activation leads to the stimulation of the parasympathetic efferent arm of the trigemino-autonomic reflex (giving rise to cranial autonomic symptoms) and also to a more frequent occurrence of central sensitization, documented by the presence of allodynia and photophobia. ${ }^{68}$

By simply reasoning in clinical terms, the trigeminal overactivation and parasympathetic stimulation during migraine attacks indicate that UAs+ have high levels of both CGRP (marker of trigeminal activation) and VIP (hallmark of parasympathetic activation).$^{10}$ This hypothesis is biochemically documented, because increased CGRP and VIP concentrations in ipsilateral jugular blood during the attack have been detected in UAs $+{ }^{69}$ Notably, UAs+ show a very convincing triptan responsiveness and manifest a sharp reduction in CGRP and VIP plasma concentrations following rizatriptan assumption. ${ }^{70-72}$ Thus, UAs+ could represent a peculiar migraine endophenotype characterized by a very good response to trigeminal-targeted treatments. ${ }^{10}$

\section{Direction of pain: exploding and imploding/ocular headache}

Directionality of pain could also matter. Migraineurs with pain pointing inward (imploding) respond better to BoNT-A prophylaxis than those with pain pointing outward (exploding), according to Jakubowski et al's findings ${ }^{73}$ In fact, pooling together the results of a prospective and of a retrospective study on a total of 63 patients affected by episodic $(n=36)$ or chronic $(\mathrm{n}=27)$ migraine, the author found that responders (i.e., individuals with a monthly migraine day reduction by $>80 \%$ compared to pre-treatment) were $100 \%(n=5)$ of patients with ocular (eye-popping) pain, $94 \%(n=29)$ of those with imploding pain and only $19 \%(n=5)$ of those with exploding pain, suggesting that an involvement of extracranial nociceptors could lie at the basis of BoNT-A responsiveness. ${ }^{73}$ Strikingly, most responders (54\%) were episodic migraineurs, in contrast to the current opinion that BoNT-A is effective only in CM. ${ }^{26,38}$ This clearly implies that pain characteristics (location and quality) are more relevant than migraine frequency in determining BoNT-A responsiveness and prompt for a more thorough clinical evaluation of migraine features of patients enrolled in pharmacological trials. ${ }^{10}$

Unfortunately, the Jakubowski et al's study did not investigate the presence of UAs, which-intriguingly-occur in the same proportion of migraineurs with imploding/ocular pain (30\%). ${ }^{68,73}$ These 2 migraine endophenotypes could indeed be coincidental, because - at least theoretically - the imploding/ ocular migraine pain could arise from extracranial nociceptor stimulation induced by periocular edema and vasodilation following the activation of the trigemino-autonomic reflex in UAs+ (Figure 2). ${ }^{68}$

\section{Duration of migraine}

Short duration of migraine, regardless of being episodic or chronic, would predict a positive response to BoNT-A treatment. This hypothesis was proposed in a prospective, openlabel study reporting a better response rate in patients with disease duration of $<30$ years, compared to those with longer migraine duration ( $>25 \%$ MIDAS score reduction: $79 \%$ vs $46 \% ; p=0.02),{ }^{74}$ suggesting that functional and biochemical brain changes due to repeated migraine episodes would render patients with longer migraine duration less sensitive to BoNT-A prophylaxis. 


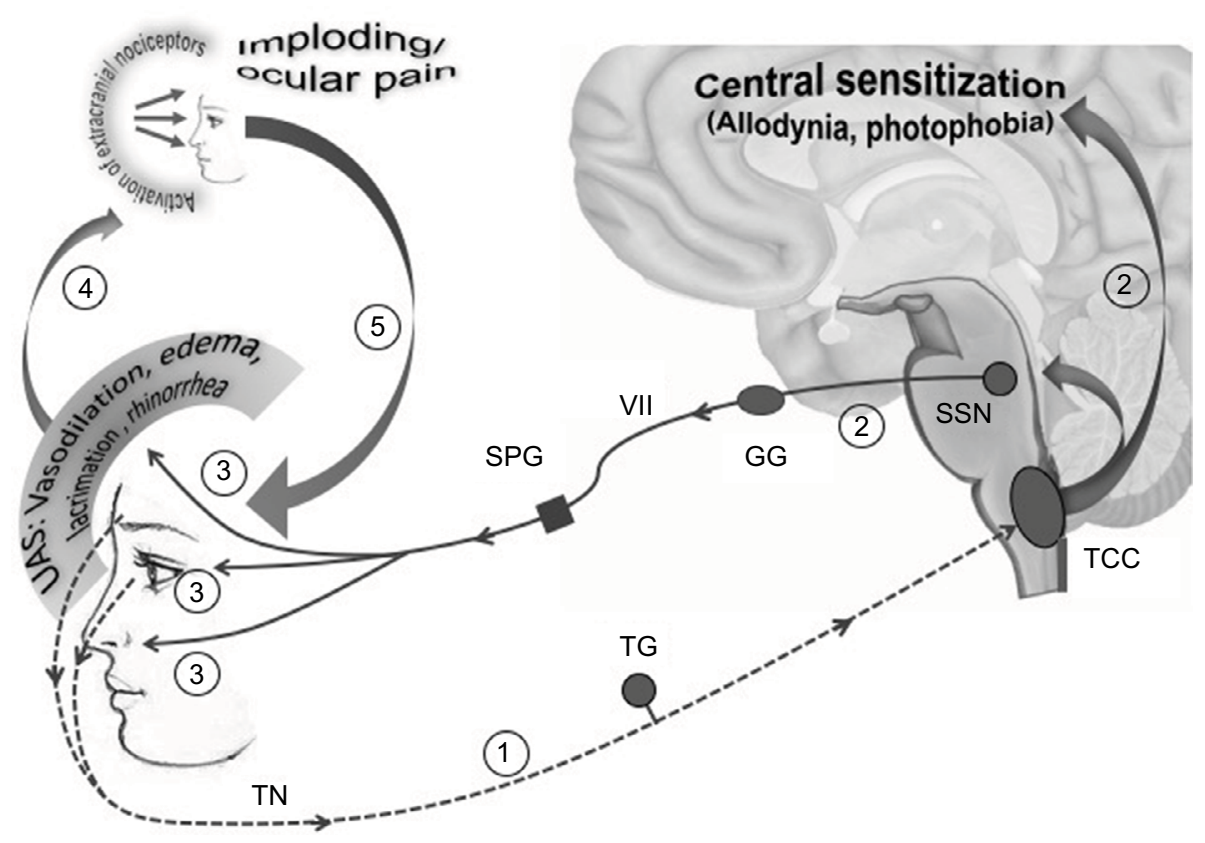

Figure 2 Imploding/ocular pain might be a consequence of the trigemino-autonomic reflex activation: a hypothesis.

Notes: In I/3 of migraineurs, an overactivation of the TN - causing a more strictly unilateral and severe headache located along the areas of cutaneous distribution of the ophthalmic branch (I) - induces both central sensitization (allodynia and photophobia) and the activation of the efferent arm of the trigeminal-autonomic reflex (2). Activated preganglionic parasympathetic fibers originating in the SSN exit the brainstem via the seventh cranial nerve (VII), traverse the GG and synapse in the SPG with postsynaptic neurons innervating cranial and conjunctival vessels, lacrimal glands and nasal mucosa, triggering UAs (ocular/periocular vasodilation and edema, lacrimation and rhinorrhea)

(3). UAs would activate extracranial nociceptors (4), being responsible for imploding/periocular pain characteristics, and would in turn amplify trigeminal afferent firing (5), further perpetuating the vicious cycle.

Abbreviations: TN, trigeminal nerve; SSN, superior salivatory nucleus; GG, geniculate ganglion; SPG, sphenopalatine; UAs, unilateral autonomic symptom; TCC, trigeminocervical complex; TG, trigeminal ganglion.

\section{Pericranial muscle tenderness}

The presence of pericranial muscle tenderness (PMT) could identify BoNT-A responders among patients with MO. A small RCT (68 patients) revealed that BoNT-A (100 U given in 16 injection sites) does not overperform placebo in terms of headache day reduction in patients affected by $\mathrm{CM}$ with $\mathrm{MO}$, inducing only a reduction in analgesic intake. When selectively considering migraineurs with PMT, however, BoNT-A was significantly more effective, reducing not only drug consumption but also headache days, pain intensity and disability measures. ${ }^{75}$ PMT, a symptom characterizing a large number of patients CTTH and reflecting a state of central nociceptive sensitization, may indeed be present in patients with CM, and is even more pronounced in CTTH with concomitant migraine. ${ }^{76}$ These data seem to indicate that BoNT-A - a drug believed to reduce peripheral and central sensitization in CM - works better when central sensitization, the hallmark of this condition, is extremely pronounced.

\section{Special considerations}

A series of clinical trials and real-life studies proved that BoNT-A represents the best option for the prophylaxis of $\mathrm{CM}$ in terms of efficacy, safety, tolerability, reduced disability and headache-related resource use, and improved quality of life..$^{43,77,78}$ Nevertheless, we deem that some major issues remain to be addressed (Table 1).

\section{BoNT-A responders' identification}

The clinical characterization of patients with $\mathrm{CM}$ in pharmacological trials with BoNT-A is unsatisfactory. Tailored therapy, a reasonable patient expectation and the goal of any effective treatment strategy, relies indeed on a careful assessment of disease clinical characteristics. The detailed assessment of individual migraine endophenotype, more than a trite application of CM classification criteria based mostly on migraine frequency, might widen BoNT-A therapeutic benefits. ${ }^{10}$ As a matter of fact, various studies outlined that unilaterality, intensity, quality and direction of pain, presence of UAs, disease duration and PMT may predict responsiveness to trigeminal targeted treatments. ${ }^{10,70,71,73-75}$ These symptoms, reflecting a higher degree of peripheral and central nociceptive sensitization in a subset of patients with $\mathrm{CM}$, might well drive the therapeutic choice of BoNT-A, a drug preferentially targeting hyperactive terminals, whose clinical efficacy is indeed related to its ability to reverse the sensitization of head pain circuitry. 
Table I BoNT-A in CM treatment: issues to be addressed

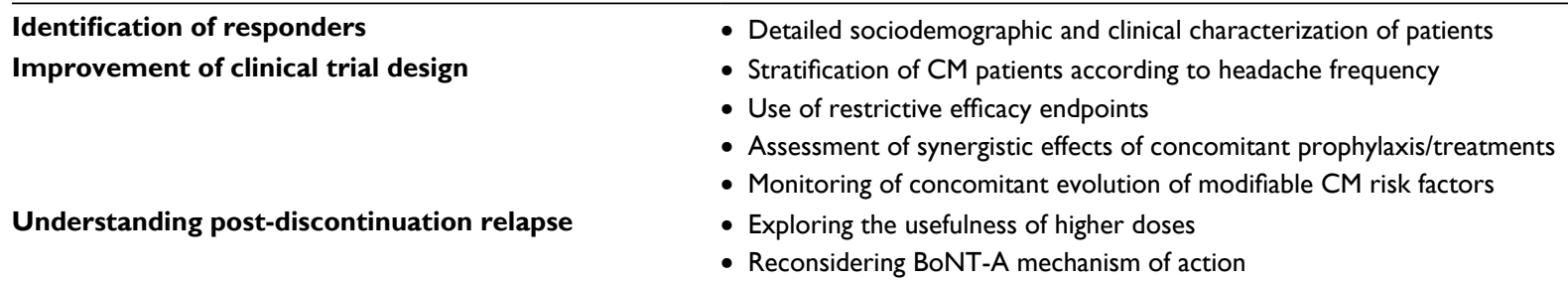

preventive agent used. Nevertheless, $\mathrm{CP}$ could, indeed, exert a positive synergistic effect with BoNT-A on CM. Hence, a stratification of patients according to the specific class of $\mathrm{CP}$ (anticonvulsants, beta-blockers, calcium-antagonists, antidepressants, etc.) would be helpful to verify whether BoNT-A produces more benefits on patients simultaneously treated with a given migraine prophylaxis. The same applies for treatments used for comorbidities (eg, depression, anxiety).

\section{Evolution of modifiable risk factors}

How to exclude that a positive clinical outcome following a long-lasting BoNT-A treatment can also be linked to reduced $\mathrm{CM}$ risk factors? ${ }^{4,80} \mathrm{CM}$ patients consulting a physician on a regular basis for the treatment cycles over 1 year or more could be more prone or motivated to concomitantly reduce modifiable CM risk factors than those treated with oral drugs, such as TPM. As a consequence, the outcome of transformation and reversion factors during trials should be monitored and taken into account in result analysis.

\section{Rapid CM relapse following BoNT-A discontinuation}

$\mathrm{CM}$ is the paradigm of a difficult-to-treat headache, given its obscure pathophysiology and complex comorbidities. However, the rapid CM relapse following BoNT-A discontinuation and, even more, the increased attack frequency when slightly postponing the subsequent treatment cycle are not easily explainable with a non-symptomatic drug capable of reverting peripheral and central sensitization, hence theoretically inducing plastic changes in pain matrix. ${ }^{54,65}$ Thus, it should be verified in future studies if this issue could be bypassed by using higher doses. Alternatively, the hypothetical BoNT-A mechanisms of action in $\mathrm{CM}$ should be reconsidered.

\section{Conclusion}

More attention to clinical migraine features and the setup of better designed clinical trials could improve the identification of BoNT-A responders, allowing a more tailored treatment for $\mathrm{CM}$. 


\section{Disclosure}

Piero Barbanti has received consultancy fees from Allergan, Bayer, electroCore and Visufarma and advisory fees from Abbott and Merck. The authors report no other conflicts of interest in this work.

\section{References}

1. Headache Classification Committee of the International Headache Society (IHS). The International Classification of Headache Disorders, 3rd edition (beta version). Cephalalgia. 2013;33(9):629-808.

2. Adams AM, Serrano D, Buse DC, et al. The impact of chronic migraine: the Chronic Migraine Epidemiology and Outcomes (CaMEO) Study methods and baseline results. Cephalalgia. 2015;35(7):563-578.

3. Bigal ME, Lipton RB. Migraine chronification. Curr Neurol Neurosci Rep. 2011;11(2):139-148.

4. Schwedt TJ. Chronic migraine. BMJ. 2014;348:g1416.

5. Boyer N, Dallel R, Artola A, Monconduit L. General trigeminospinal central sensitization and impaired descending pain inhibitory controls contribute to migraine progression. Pain. 2014;155(7):1196-1205.

6. de Tommaso M, Valeriani M, Guido M, et al. Abnormal brain processing of cutaneous pain in patients with chronic migraine. Pain. 2003;101(1-2):25-32.

7. Perrotta A, Serrao M, Sandrini G, et al. Sensitisation of spinal cord pain processing in medication overuse headache involves supraspinal pain control. Cephalalgia. 2010;30(3):272-284.

8. Coppola G, Schoenen J. Cortical excitability in chronic migraine. Curr Pain Headache Rep. 2012;16(1):93-100.

9. Escher CM, Paracka L, Dressler D, Kollewe K. Botulinum toxin in the management of chronic migraine: clinical evidence and experience. Ther Adv Neurol Disord. 2017;10(2):127-135.

10. Barbanti P, Egeo G. Pharmacological trials in migraine: it's time to reappraise where the headache is and what the pain is like. Headache. 2015;55(3):439-441.

11. Erbguth FJ. From poison to remedy: the chequered history of botulinum toxin. J Neural Transm (Vienna). 2008;115(4):559-565.

12. Matak I, Lacković Z. Botulinum toxin A, brain and pain. Prog Neurobiol. 2014;119-120:39-59.

13. Martin TF. Stages of regulated exocytosis. Trends Cell Biol. 1997;7(7):271-276.

14. Binder WJ, Blitzer A, Brin MF. Treatment of hyperfunctional lines of the face with botulinum toxin A. Dermatol Surg. 1998;24(11):1198-1205.

15. Silberstein SD, Göbel H, Jensen R, et al. Botulinum toxin type A in the prophylactic treatment of chronic tension-type headache: a multicentre, double-blind, randomized, placebo-controlled, parallel-group study. Cephalalgia. 2006;26(7):790-800.

16. Mathew NT, Frishberg BM, Gawel M, Dimitrova R, Gibson J, Turkel C; BOTOX CDH Study Group. Botulinum toxin type A (BOTOX) for the prophylactic treatment of chronic daily headache: a randomized, double-blind, placebo-controlled trial. Headache. 2005;45(4):293-307.

17. Blumenfeld AM, Binder W, Silberstein SD, Blitzer A. Procedures for administering botulinum toxin type A for migraine and tension-type headache. Headache. 2003;43(8):884-891.

18. Silberstein SD, Lipton RB. Chronic daily headache. Curr Opin Neurol. 2000;13(3):277-283

19. Binder WJ, Brin MF, Blitzer A, Schoenrock LD, Pogoda, JM. Botulinum toxin type A (BOTOX) for treatment of migraine headaches: an open-label study. Otolaryngol Head Neck Surg. 2000;123(6):669-676.

20. Shuhendler AJ, Lee S, Siu M, et al. Efficacy of botulinum toxin type A for the prophylaxis of episodic migraine headaches: a meta-analysis of randomized, double-blind, placebo-controlled trials. Pharmacotherapy. 2009;29(7):784-791.

21. Sostak P, Krause P, Förderreuther S, Reinisch V, Straube A. Botulinum toxin type-A therapy in cluster headache: an open study. $J$ Headache Pain. 2007;8(4):236-241.
22. Bratbak DF, Nordgård S, Stovner LJ, et al. Pilot study of sphenopalatine injection of onabotulinumtoxinA for the treatment of intractable chronic cluster headache. Cephalalgia. 2016;36(6):503-509.

23. Miller S, Correia F, Lagrata S, Matharu MS. OnabotulinumtoxinA for hemicrania continua: open label experience in 9 patients. $J$ Headache Pain. 2015;16:19.

24. Volcy M, Tepper SJ, Rapoport AM, Sheftell FD, Bigal ME. Botulinum toxin A for the treatment of greater occipital neuralgia and trigeminal neuralgia: a case report with pathophysiological considerations. Cephalalgia. 2006;26(3):336-340.

25. Kapural L, Stillman M, Kapural M, McIntyre P, Guirgius M, Mekhail N. Botulinum toxin occipital nerve block for the treatment of severe occipital neuralgia: a case series. Pain Pract. 2007;7(4):337-340.

26. Taylor M, Silva S, Cottrell C. Botulinum toxin type-A (BOTOX) in the treatment of occipital neuralgia: a pilot study. Headache. 2008;48(10): 1476-1481.

27. Morra ME, Elgebaly A, Elmaraezy A, et al. Therapeutic efficacy and safety of Botulinum Toxin A Therapy in Trigeminal Neuralgia: a systematic review and meta-analysis of randomized controlled trials. J Headache Pain. 2016;17(1):63.

28. Headache Classification Subcommittee of the International Headache Society. The International Classification of Headache Disorders: 2nd edition. Cephalalgia. 2004;24(Supp1 1):9-160.

29. Headache Classification Committee, Olesen J, Bousser MG, Diener $\mathrm{HC}$, et al. New appendix criteria open for a broader concept of chronic migraine. Cephalalgia. 2006;26(6):742-746.

30. Aurora SK, Dodick DW, Turkel CC, et al; PREEMPT 1 Chronic Migraine Study Group. OnabotulinumtoxinA for treatment of chronic migraine: results from the double-blind, randomized, placebo-controlled phase of the PREEMPT 1 trial. Cephalalgia. 2010;30(7):793-803.

31. Diener HC, Dodick DW, Aurora SK, et al; PREEMPT 2 Chronic Migraine Study Group. OnabotulinumtoxinA for treatment of chronic migraine: results from the double-blind, randomized, placebo-controlled phase of the PREEMPT 2 trial. Cephalalgia. 2010;30(7):804-814.

32. Aurora SK, Winner P, Freeman MC, et al. OnabotulinumtoxinA for treatment of chronic migraine: pooled analyses of the 56-week PREEMPT clinical program. Headache. 2011;51(9):1358-1373.

33. Diener HC, Dodick DW, Turkel CC, et al. Pooled analysis of the safety and tolerability of onabotulinumtoxinA in the treatment of chronic migraine. Eur J Neurol. 2014;21(6):851-859.

34. Silberstein SD, Dodick DW, Aurora SK, et al. Per cent of patients with chronic migraine who responded per onabotulinumtoxinA treatment cycle: PREEMPT. J Neurol Neurosurg Psychiatry. 2015;86(9):996-1001.

35. Silberstein SD, Blumenfeld AM, Cady RK, et al. OnabotulinumtoxinA for treatment of chronic migraine: PREEMPT 24-week pooled subgroup analysis of patients who had acute headache medication overuse at baseline. J Neurol Sci. 2013;331(1-2):48-56.

36. Aurora SK, Dodick DW, Diener HC, et al. OnabotulinumtoxinA for chronic migraine: efficacy, safety, and tolerability in patients who received all five treatment cycles in the PREEMPT clinical program. Acta Neurol Scand. 2014;129(1):61-70.

37. Lipton RB, Rosen NL, Ailani J, DeGryse RE, Gillard PJ, Varon SF. OnabotulinumtoxinA improves quality of life and reduces impact of chronic migraine over one year of treatment: pooled results from the PREEMPT randomized clinical trial program. Cephalalgia. 2016;36(9):899-908.

38. Santoro A, Tanzi M, Miscio A, Copetti M, Leone M. P025. Two-year follow-up with OnabotulinumtoxinA for chronic migraine: a real-life evaluation of 113 patients. J Headache Pain. 2015;16(Suppl 1):A182.

39. Pedraza MI, de la Cruz C, Ruiz M, et al. OnabotulinumtoxinA treatment for chronic migraine: experience in 52 patients treated with the PREEMPT paradigm. Springerplus. 2015;4:176.

40. Grazzi L, Usai S. Onabotulinum toxin A (Botox) for chronic migraine treatment: an Italian experience. Neurol Sci. 2015;36(Suppl 1):33-35.

41. Kollewe K, Escher CM, Wulff DU, et al. Long-term treatment of chronic migraine with OnabotulinumtoxinA: efficacy, quality of life and tolerability in a real-life setting. $J$ Neural Transm (Vienna). 2016;123(5):533-540. 
42. Aicua-Rapun I, Martínez-Velasco E, Rojo A, et al. Real-life data in 115 chronic migraine patients treated with onabotulinumtoxin A during more than one year. J Headache Pain. 2016;17(1):112.

43. Russo M, Manzoni GC, Taga A, et al. The use of onabotulinum toxin A (Botox $(\circledR))$ in the treatment of chronic migraine at the Parma Headache Centre: a prospective observational study. Neurol Sci. 2016;37(7): 1127-1131.

44. Vikelis M, Argyriou AA, Dermitzakis EV, Spingos KC, Mitsikostas DD. Onabotulinumtoxin-A treatment in Greek patients with chronic migraine. J Headache Pain. 2016;17(1):84.

45. Khalil M, Zafar HW, Quarshie V, Ahmed F. Prospective analysis of the use of OnabotulinumtoxinA (BOTOX) in the treatment of chronic migraine; real-life data in 254 patients from Hull, U.K. J Headache Pain. 2014;15:54.

46. Ahmed F, Zafar HW, Buture A, Khalil M. Does analgesic overuse matter? Response to OnabotulinumtoxinA in patients with chronic migraine with or without medication overuse. SpringerPlus. 2015;4:589.

47. Cernuda-Morollón E, Ramón C, Larrosa D, Alvarez R, Riesco N, Pascual J. Long-term experience with onabotulinumtoxinA in the treatment of chronic migraine: What happens after one year? Cephalalgia. 2015;35(10):864-868.

48. Guerzoni S, Pellesi L, Baraldi C, Pini LA. Increased efficacy of regularly repeated cycles with Onabotulinumtoxin $\mathrm{A}$ in $\mathrm{MOH}$ patients beyond the first year of treatment. J Headache Pain. 2015;17:48.

49. Negro A, Curto M, Lionetto L, Martelletti P. A two years open-label prospective study of OnabotulinumtoxinA $195 \mathrm{U}$ in medication overuse headache: a real-world experience. J Headache Pain. 2015;17:1.

50. Grazzi L. Onabotulinumtoxin A for chronic migraine with medication overuse: clinical results of a long-term treatment. Neurol Sci. 2017;38(Suppl 1):141-143.

51. Diener HC, Dodick DW, Goadsby PJ, Lipton RB, Olesen J, Silberstein SD. Chronic migraine--classification, characteristics and treatment. Nat Rev Neurol. 2012;8(3):162-171.

52. Mathew NT, Jaffri SF. A double-blind comparison of onabotulinumtoxina (BOTOX) and topiramate (TOPAMAX) for the prophylactic treatment of chronic migraine: a pilot study. Headache. 2009;49(10): 1466-1478.

53. Cady RK, Schreiber CP, Porter JA, Blumenfeld AM, Farmer KU. A multi-center double-blind pilot comparison of onabotulinumtoxinA and topiramate for the prophylactic treatment of chronic migraine. Headache. 2011;51(1):21-32.

54. Boudreau GP, Grosberg BM, McAllister PJ, Lipton RB, Buse DC. Prophylactic onabotulinumtoxinA in patients with chronic migraine and comorbid depression: An open-label, multicenter, pilot study of efficacy, safety and effect on headache-related disability, depression, and anxiety. Int J Gen Med. 2015;8:79-86.

55. Barbanti P, Fabbrini G, Pauletti C, Defazio G, Cruccu G, Berardelli A. Headache in cranial and cervical dystonia. Neurology. 2005;64(7): 1308-1309

56. Winner PK, Sadowsky CH, Martinez WC, Zuniga JA, Poulette A. Concurrent onabotulinumtoxinA treatment of cervical dystonia and concomitant migraine. Headache. 2012;52(8):1219-1225.

57. Whitcup SM, Turkel CC, DeGryse RE, Brin MF. Development of onabotulinumtoxinA for chronic migraine. Ann N Y Acad Sci. 2014;1329:67-80.

58. Aoki KR. Review of a proposed mechanism for the antinociceptive action of botulinum toxin type A. Neurotoxicology. 2005;26(5):785-793.

59. Aoki KR, Francis J. Updates on the antinociceptive mechanism hypothesis of botulinum toxin A. Parkinsonism Relat Disord. 2011;17(Suppl 1): S28-S33.

60. Dolly JO, Aoki KR. The structure and mode of action of different botulinum toxins. Eur J Neurol. 2006;13(Suppl 4):1-9.

61. Shimizu T, Shibata M, Toriumi H, et al. Reduction of TRPV1 expression in the trigeminal system by botulinum neurotoxin type-A. Neurobiol Dis. 2012;48(3):367-378.
62. Mazzocchio R, Caleo M. More than at the neuromuscular synapse: actions of botulinum neurotoxin $\mathrm{A}$ in the central nervous system. Neuroscientist. 2015;21(1):44-61.

63. Hubbard CS, Becerra L, Smith JH, et al. Brain changes in responders vs. non-responders in chronic migraine: markers of disease reversal. Front Hum Neurosci. 2016;10:497. eCollection 2016.

64. Cernuda-Morollón E, Martínez-Camblor P, Ramón C, Larrosa D, Serrano-Pertierra E, Pascual J. CGRP and VIP levels as predictors of efficacy of Onabotulinumtoxin type A in chronic migraine. Headache. 2014;54(6):987-995.

65. Cernuda-Morollón E, Ramón C, Martínez-Camblor P, SerranoPertierra E, Larrosa D, Pascual J. OnabotulinumtoxinA decreases interictal CGRP plasma levels in patients with chronic migraine. Pain. 2015;156(5):820-824.

66. Cernuda-Morollón E, Larrosa D, Ramón C, Vega J, Martínez-Camblor $\mathrm{P}$, Pascual J. Interictal increase of CGRP levels in peripheral blood as a biomarker for chronic migraine. Neurology. 2013;81(14):1191-1196.

67. Barbanti P, Fabbrini G, Pesare M, Vanacore N, Cerbo R. Unilateral cranial autonomic symptoms in migraine. Cephalalgia. 2002;22(4):256-259.

68. Barbanti P, Aurilia C, Dall'Armi V, Egeo G, Fofi L, Bonassi S. The phenotype of migraine with unilateral cranial autonomic symptoms documents increased peripheral and central trigeminal sensitization. A case series of 757 patients. Cephalalgia. 2016;36(14):1334-1340.

69. Goadsby PJ, Edvinsson L, Ekman R. Vasoactive peptide release in the extracerebral circulation of humans during migraine headache. Ann Neurol. 1990;28(2):183-187.

70. Barbanti P, Fabbrini G, Vanacore N, Pesare M, Buzzi MG. Sumatriptan in migraine with unilateral cranial autonomic symptoms: an open study. Headache. 2003;43(4):400-403.

71. Barbanti P, Fofi L, Dall'Armi V, et al. Rizatriptan in migraineurs with unilateral cranial autonomic symptoms: a double-blind trial. J Headache Pain. 2012;13(5):407-414.

72. Sarchielli P, Pini LA, Zanchin G, et al. Clinical-biochemical correlates of migraine attacks in rizatriptan responders and non-responders. Cephalalgia. 2006;26(3):257-265.

73. Jakubowski M, McAllister PJ, Bajwa ZH, Ward TN, Smith P, Burstein R. Exploding vs. imploding headache in migraine prophylaxis with Botulinum Toxin A. Pain. 2006;125(3):286-295.

74. Eross EJ, Gladstone JP, Lewis S, Rogers R, Dodick DW. Duration of migraine is a predictor for response to botulinum toxin type A. Headache. 2005;45(4):308-314.

75. Sandrini G, Perrotta A, Tassorelli C, et al. Botulinum toxin type-A in the prophylactic treatment of medication-overuse headache: a multicenter, double-blind, randomized, placebo-controlled, parallel group study. J Headache Pain. 2011;12(4):427-433.

76. Aaseth K, Grande RB, Lundqvist C, Russell MB. Pericranial tenderness in chronic tension-type headache: the Akershus population-based study of chronic headache. J Headache Pain. 2014;15:58.

77. Lipton RB, Varon SF, Grosberg B, et al. OnabotulinumtoxinA improves quality of life and reduces impact of chronic migraine. Neurology. 2011;77(15):1465-1472.

78. Hepp Z, Rosen NL, Gillard PG, Varon SF, Mathew N, Dodick DW. Comparative effectiveness of onabotulinumtoxinA versus oral migraine prophylactic medications on headache-related resource utilization in the management of chronic migraine: retrospective analysis of a US-based insurance claims database. Cephalalgia. 2016;36(9):862-874.

79. Scher AI, Midgette LA, Lipton RB. Risk factors for headache chronification. Headache. 2008;48(1):16-25.

80. Manack A, Buse DC, Serrano D, Turkel CC, Lipton RB. Rates, predictors, and consequences of remission from chronic migraine to episodic migraine. Neurology. 2011;76(8):711-718.

81. Manzoni GC, Bonavita V, Bussone G, et al; ANIRCEF (Associazione Neurologica Italiana Ricerca Cefalee). Chronic migraine classification: current knowledge and future perspectives. J Headache Pain. 2011;12(6):585-592. 
The Journal of Pain Research is an international, peer reviewed, open access, online journal that welcomes laboratory and clinical findings in the fields of pain research and the prevention and management of pain. Original research, reviews, symposium reports, hypothesis formation and commentaries are all considered for publication
The manuscript management system is completely online and includes a very quick and fair peer-review system, which is all easy to use. Visit http://www.dovepress.com/testimonials.php to read real quotes from published authors. 